\title{
Reliability of Leukocytosis in Diagnosing Acute Appendicitis
}

\author{
Vikram Panjabrao Vaidya ${ }^{1}$, Yashwant R. Lamture², Harshal Ramteke³, Aditya Mundada4, \\ Varsha Gajbhiye $^{5}$, Minakshi Yeola ${ }^{6}$
}

\begin{abstract}
${ }^{1}$ Department of Surgery, Jawaharlal Nehru Medical College, Wardha, Maharashtra, India. ${ }^{2}$ Department of Surgery, Datta Meghe Medical College, Nagpur, Maharashtra, India. ${ }^{3}$ Department of Surgery, Jawaharlal Nehru Medical College, Wardha, Maharashtra, India. ${ }^{4}$ Department of Surgery, Jawaharlal Nehru Medical College, Wardha, Maharashtra, India. ${ }^{5}$ Department of Pharmacology, Datta Meghe Medical College, Nagpur, Maharashtra, India. ${ }^{6}$ Department of Surgery, Jawaharlal Nehru Medical College, Wardha, Maharashtra, India.
\end{abstract}

\section{ABSTRACT}

\section{BACKGROUND}

Acute appendicitis is a frequently encountered surgical disease. Detection is difficult even with imaging and blood investigations. Total leukocyte count is a promising investigation. Its role in the confirmation of acute appendicitis is investigated and analysed in the present study.

\section{METHODS}

Patients having acute appendicitis treated by surgical intervention (appendicectomy) were included in the study. The leukocyte count measured before surgery was compared with histopathology findings of the appendix. Parameters of diagnostic accuracy of leukocytosis were measured by standard formulae.

\section{RESULTS}

The sensitivity and specificity of leucocytosis were found to be 76 percent and 12.5 percent respectively. The positive predictive value and negative predictive value of leucocytosis was 65 percent and 20 percent respectively. Overall diagnostic accuracy was 56 percent. The results were compared with histopathology of acute appendicitis.

\section{CONCLUSIONS}

Leukocytosis is a helpful investigation to support the diagnosis of acute appendicitis.

\section{KEY WORDS}

Leukocytosis, Gangrene of Appendix, Negative Appendectomy
Corresponding Author:

Dr. Yashwant R. Lamture,

Professor,

Department of Surgery,

Datta Meghe Medical College,

Nagpur, Maharashtra, India.

E-mail:yash18671@gmail.com

DOI: $10.14260 /$ jemds/2020/493

How to Cite This Article:

Vaidya VP, Lamture YR, Ramteke $H$, et al. Reliability of leukocytosis in diagnosing acute appendicitis. J. Evolution Med. Dent. Sci. 2020;9(32):2274-2278, DOI: $10.14260 /$ jemds/2020/493

Submission 24-04-2020,

Peer Review 01-07-2020,

Acceptance 07-07-2020,

Published 10-08-2020.

Copyright (C) 2020 JEMDS. This is an open access article distributed under Creative Commons Attribution License [Attribution 4.0 International (CC BY 4.0)] 


\section{BACKGROUND}

Acute Appendicitis is a common aetiology of pain abdomen in all ages. It contributes to around $10 \%$ of laparotomies and onethird of all acute abdominal pain in children. It is a difficult job for a clinician to correctly diagnose acute appendicitis. Numerous scores and investigations like C-reactive proteins (CRP) are more confusing for the surgeon as these are not $100 \%$ correct. Total Leucocyte count (TLC) is a useful investigation for acute appendicitis. ${ }^{1}$ Leukocytosis, most of the time less than 18,000 is present in patients with acute, uncomplicated appendicitis. TLC is cheap and available in almost all primary health centres. Numerous studies are published on the role of Leucocytosis in acute appendicitis. The diagnostic accuracy of TLC is increased further if combined with other markers of inflammation. ${ }^{2}$

Acute appendicitis is a common surgical disease with a lifetime incidence of $6 \%$ and most affected somewhat between 11-32 years of age but can occur at any age. A male is more commonly affected than females. The overall lifetime risk is 8.4\% for males and 6.8\% for females in the America. Around 3 lakh appendectomies are done in the United States each year and more than 40,000 cases of acute appendicitis are admitted to the surgery department in the united kingdom per year. While uncomplicated acute appendicitis is having less than $1 \%$ death rate, this increases to $5 \%$ or more for younger children and old patients due to the more possibility of perforation and complications due to late diagnosis. Even today the ideal option for treatment of appendicitis is appendectomy. As early treatment of appendicitis is required to decrease morbidity and deaths, certain relaxations of making overdiagnosis is agreed by most of the surgical schools. An aggressive treatment by the surgeon for doubtful patients of acute appendicitis (i.e., overdiagnosis) will lead to more chances of negative laparotomy; whereas a conservative treatment (i.e., under-diagnosis) will cause a higher rate of perforation and other complications. Hence the treating physician is having a difficult task of making correct equilibrium between overdiagnosis and under diagnosis. Here, diagnosis supportive agents could be of great importance by helping the surgeon to operate and remove appendix early and thereby decreases both negative laparotomy rate and incidence of perforations and deaths. However, these diagnosis supportive agents come with their group of drawbacks and these are discussed below. While the significance of diagnostic laparoscopy has proved to decrease the chances of negative laparotomy by around $25 \%$ and may be useful in doubtful cases, especially in women of reproductive age group or in with high body mass index; but it should be used only after all other methods and investigations, as it is not non-invasive intervention and has associated potential of infection and complications. The significance of diagnostic imaging for doubtful cases of acute appendicitis has improved in modern years. But, Ultrasonography (USG) and Computerized Tomography (CT) cannot be used without the aim for all cases having right-sided abdominal pain because these methods are costly, not available in all hospital and have disadvantage of ionizing radiations (e.g., CT) and operator dependent diagnostic ability (e.g., USG). Few studies suggested that USG and CT scanning in the diagnosis of acute appendicitis, should be done only in those patients who have a difficulty in diagnosis of appendicitis by clinical and laboratory tests. As USG is having a disadvantage of operator dependency and needs experienced person, it is preferable to use CT to USG, as CT has greater diagnostic accuracy. CT scan and USG both are used to assess patients with a doubtful diagnosis of appendicitis. As already stated CT scan has better sensitivity and specificity in comparison to USG with sensitivity and specificity. Still bad part is that both imaging modalities are not able to reduce the negative appendectomy rate. As rural Indian hospitals lack the availability of modern imaging studies and manpower. Patients are usually underdiagnosed or overdiagnosed and based on guesswork, they are referred to higher centers for treatment. This is costly, as patients spend a lot of (i) valuable time to reach higher centers and in waiting for the specialists and (ii) money in traveling and maintenance. ${ }^{3}$

A negative appendectomy is considered if histopathology specimen reveal normal. many methods have been invented to help in doubtful cases to decrease negative appendectomy rates (NAR). Several scorings have been devised for improving early diagnosis of acute appendicitis and its appropriate treatment. These scores uses clinical history, physical examination and laboratory findings. The diagnosis of acute appendicitis is based on history, clinical examination and a few laboratory investigations. In all patients, but, a definitive diagnosis can only possible after histopathological evaluation of the surgical specimen. Before operation the diagnostic correctness of acute appendicitis remains poor, ranging from $25 \%$ to $80 \%$ and more difficult in females gender than in males. Also a NAR of $21-41 \%$ has been verified and many physician consider a rate of $30 \%$ as not possible to prevent. Removal of a normal appendix is an economic loss to both the patients and hospital. in an attempt to avoid it a can lead to incorrect diagnosis and delay in surgery with possibility of complications like perforation and peritonitis. ${ }^{4}$

Though typical without complication patients of acute appendicitis are easy to identify and treat. But in atypical cases of it is a hard job and a difficult challenge to even experienced clinician. Even with improvement in the diagnostic and scanning methods, the negative appendicectomy rates have not decreased much. Clinical examination is still the most guiding tool in the treatment of cases with doubtful acute appendicitis. Use of CT scan or diagnostic laparoscopy for all patients who are suspected to have acute appendicitis is not cost-effective safe. But, if used in a selected suspected patients can improve the diagnostic accuracy and decreases the cost and rate of negative appendicectomy. ${ }^{5}$

The identification of acute appendicitis is still hard and may be the not uncommon diagnostic dilemma in surgical fraternity. The typical combination of three elements, a reliable history, pain at Mc Burney's point and leukocytosis has a diagnostic accuracy of $<80 \%$. Along with these, if ultrasonography, computerized topography or radionuclide scanning added, it can improve not above $90 \%$. The strategy of early surgery has been not uncommon for many years but may cause a large number of normal appendices being removed. Some surgeon advised that the method of "operate and diagnose" is better than "wait and observe" as an unnecessary surgery is better than an unaccepted perforation. As surgery itself may have complications (10-15\%) as wound infection, deep vein thrombosis, chest infection, adhesive bowel obstruction etc. increased levels of Total leukocyte count (TLC) with clinical signs and symptoms favours to possibility of acute appendicitis but it may to have even perforated 
appendix with normal TLC and differential leukocyte count. C reactive protein is the prototype acute phase reactant, produced by liver, its level increases in 8 hours of start of injury/inflammation of tissue, reaches maximum within 24 48 hours and remains at high as long as continuing tissue inflammation or destruction. Regular measurement of TLC and CRP in suspected appendicitis may improve accuracy of diagnosing acute appendicitis. ${ }^{6}$

Every year we find in the medical literature new tests related to inflammatory markers to confirm the diagnosis of acute appendicitis, and in many of these studies the investigators always mention the white blood cell count (WBC) which indicates that this is an important reference test that, in addition, has the great advantage of being available in all health facilities.

The most common biological markers that have been studied in the diagnosis of acute appendicitis, apart from the WBC, are: The Differential Leukocyte Count (DLC), the CReactive Protein (CRP), Erythrocyte Sedimentation Reaction (ESR), Tumour Necrosis Alpha (TNF-alpha), Alpha 1Glycoprotein (Alpha 1gp), leukocyte elastase complex (Elastase), Interleukin-8 (IL-8), Interleukin-6 (IL-6), Interleukin-10 (IL-10), granulocyte colony stimulating factor, interferon gamma, soluble intercellular adhesion molecule-1, matrix metalloproteinase-9, tissue inhibitor metalloproteinase-1, serum amyloid A, plasma calprotectin, plasma serotonin serum Leucine-Rich Alpha Glycoprotein-1 (LRG), and procalcitonin However, none of these tests have been proved to be useful in the early diagnosis of acute appendicitis. Furthermore, all of these markers are nonspecific because what they are measuring is the grade of an inflammatory process. For instance, according to Jangioo, the most used inflammatory marker, the C-reactive protein, is not an ideal diagnostic tool for ruling out or for determination of acute appendicitis. Pruekprasert et al. compared the surgeon's clinical diagnosis of acute appendicitis with the Alvarado Score and measurement of the CRP, and demonstrated that the sensitivity of the surgeon's assessment was $96 \%$ versus $79 \%$ for the Alvarado score and 62\% for the CRP. The accuracy for the surgeon's assessment, the Alvarado score, and the CRP were $90 \%, 72 \%$, and $61 \%$, respectively. Atema, in a study to evaluate the accuracy of the WBC and C-reactive protein in relation to the duration of symptoms in patients suspected of acute appendicitis, found that none of these tests can safely and sufficiently confirm or exclude the diagnosis of acute appendicitis. They concluded that, in patients suspected of acute appendicitis, normal inflammatory markers could not "rule out" acute appendicitis, regardless of the duration of symptoms. Based on these findings, they could not support the use of these biomarkers, either in isolation or in combination, to confirm or exclude a diagnosis of acute appendicitis. ${ }^{7}$

The Birchley $\mathrm{D}^{8}$ explains that individual history, clinical signs, and blood investigations are feeble predictors of acute inflammation of appendix' However if they are combined, they give better diagnostic value. Hence tests like TLC and CRP are more potent in combination.

A clinically decided appendectomy has a net negative laparotomy rate ranges from $15 \%$ to $30 \%$. Hence in an attempt to reduce the removal of normal appendices should not lead to an increase in the number of perforations. ${ }^{9}$ Appendicectomy is not without complications, it may cause wound infection (10$15 \%)$, adhesive obstruction, thromboembolism, pulmonary infection, etc. Leukocytosis in combination with clinical examination can correctly diagnose acute appendicitis but sometimes it was depicted in a few studies that even perforated appendix has normal TLC and differential leukocyte count. 6

We wanted to evaluate the role of leucocytosis in diagnosing acute appendicitis.

\section{METHODS}

The present study was undertaken in the department of surgery, Datta Meghe Medical College, Hingana, Nagpur, in collaboration with Jawaharlal Nehru Medical College, Datta Meghe Institute of Medical Science, Sawangi, Meghe, Wardha, Maharashtra, India. This one is a prospective observational study. The duration of this study was from January 2019 to January 2020. The numbers of cases studied were 50 .

\section{Ethical Considerations}

The study was conducted after getting clearance from the Medical Ethical Committee of the Datta Meghe institute of medical sciences deemed university Nagpur. Only patients who gave written informed consent were included in the study.

\section{Sample Size}

The sample size was calculated as per formula was around 48 . Hence 50 sample size was sufficient $(\mathrm{N}=2(\mathrm{Z} \alpha+\mathrm{Z} 1-\beta) 2 \sigma 2 / \Delta 2)$

\section{Inclusion Criteria}

Cases of acute Appendicitis operated for appendicectomy. (All patients operated as per diagnosis by a qualified consultant in surgery department)

\section{Exclusion Criteria}

1. Pregnancy.

2. Appendicular mass or abscess.

3. Urinary calculi and gynaecological diseases.

\begin{abstract}
Statistical Analysis
The data collected included the presenting complaints, clinical signs, laboratory investigations (white cell count more than 10000) and ultrasonography. The sensitivity, specificity, positive predictive value (PPV), negative predictive value (NPV) and diagnostic accuracy of TLC calculated by using SPSS 17.0 statistical software. Ethical approval for the study was obtained from the ethics committee of DMIMS University.
\end{abstract}

\section{RESULTS}

Around 50 patients included in this prospective study. 32 males and 18 females were present. Age ranges from 18 to 58 
years and most of the cases in 20 to 30 years (40\%).

Out of 50 patients 40 patients present with leucocytosis. 26 patients correctly correlate with acute appendicitis. Remaining 14 patients do not have acute appendicitis on histopathology. Out of 10 patients without leucocytosis, only two patients have normal appendix rest 8 patients have evidence of acute appendicitis. A total of 34 patients have evidence of acute appendicitis on histopathology and 16 patients revealed normal appendices. Hence the net negative appendectomy rate was $16 \%$.

The sensitivity and specificity of leucocytosis were found to be 76 percent and 12.5 percent respectively. The positive predictive value and negative predictive value of leucocytosis was 65 percent and 20 percent respectively. Overall diagnostic accuracy was 56 percent.

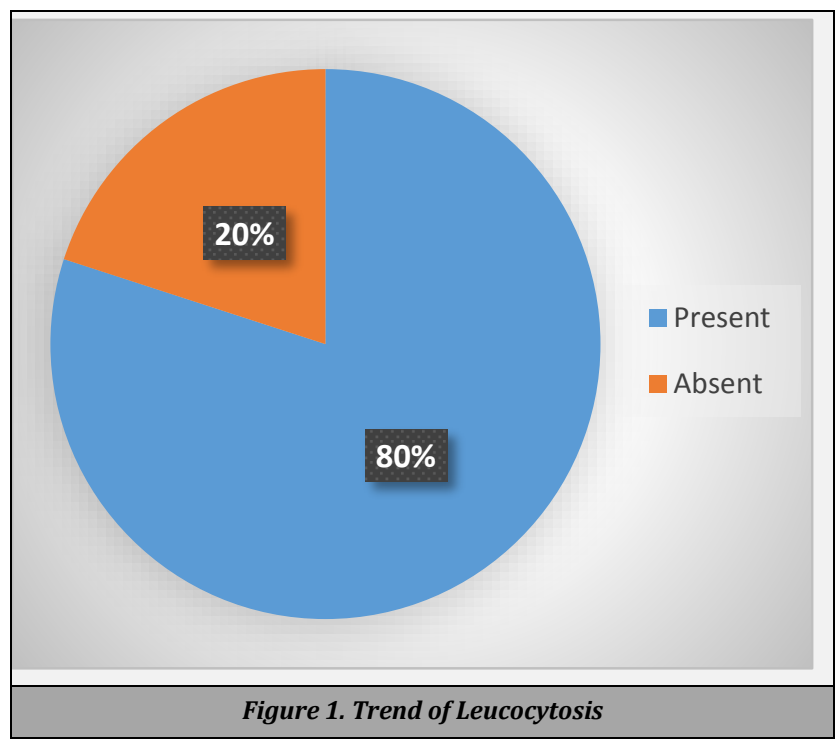

\begin{tabular}{|ccccc|}
\hline Sensitivity & Specificity & PPV & NPV & Diagnostic Accuracy \\
$76 \%$ & $12.5 \%$ & $65 \%$ & $20 \%$ & $58 \%$ \\
\hline \multicolumn{4}{|c}{ Table 1. Diagnostic Accuracy of Leucocytosis } \\
When Compared with Histopathology (Gold Standard Test) \\
\hline
\end{tabular}

\section{DISCUSSION}

Overall diagnostic accuracy of leucocytosis was $56 \%$ in the present study with a low negative predictive value of $20 \%$. Sensitivity was towards the higher side (Figure 1, and table 1). As already stated by the Birchley $D^{3}$ that in combination, leucocytosis can provide high diagnostic power. So, it is an integral part of most of the scoring systems. ${ }^{9}$

Some researchers have verified the role of inflammatory markers with variable interpretations. The sensitivity and specificity of TLC as calculated in a study conducted by Haider Kamran et al ${ }^{1}$ is $76.5 \%$ and $73.7 \%$ respectively while the positive predictive value is $92.5 \%{ }^{1}$. These results were better than the present study.

Salman Y. et $\mathrm{al}^{2}$ suggests in his study that a leucocytosis is a reliable indicator of the severity of appendicitis and signifies a more advanced stage. Out of a total of 232 patients, leucocytosis was $71.9 \%$ and normal in $28.1 \%$ c. Mean leucocyte count in acute appendicitis was 14500, in a presence of gangrene was 17100 and with perforated appendicitis was 17900 . This shows a higher total leucocyte count in complicated appendicitis. In the present study in $80 \%$ of patients have leucocytosis. Mean leucocyte count also shows an increasing trend in complicated appendicitis,

Ahmad Q.A., et $\mathrm{al}^{6}$ in his study shows a positive predictive value of both CRP and TLC in combination was around $100 \%$. It is more than that of individual parameters (CRP 100\% and TLC 93\%). The negative predictive value of a combination of both TLC and CRP (66\%) was better than individual TLC (50\%) and CRP (50\%). however, the results were superior to the present study.

Sang Hyun Haa et $\mathrm{al}^{10}$ studied 674 children of acute abdominal pain with fever and found to have appendicitis in 119 patients. The mean total leukocyte count was 14800 in acute appendicitis. This level of TLC was significantly higher than patients without appendicitis (mean TLC was 9700). The results of the present study were almost similar (mean TLC was 14000 in the appendicitis group and 8200 in nonappendicitis groups).

A study was conducted by Saaiq $\mathrm{M}$ et al ${ }^{11}$ revealed, sensitivity, specificity, positive predictive value and negative predictive value of leucocytosis were $91 \%, 43 \%, 81 \%$, and $65 \%$ respectively. The study also shows a net negative appendectomy rate (NAR) of $17 \%$. The present study also reveals NAR of $16 \%$.

A study conducted by Al-Gaithy et al 12 shows that TLC was higher in complicated appendicitis. Leukocytosis had higher diagnostic accuracy. Sensitivity and specificity were $76 \%$ and $65 \%$ respectively. Positive predictive value (PPV) and negative predictive value (NPV) were $97 \%$ and $16 \%$ respectively, Authors further suggest that increased TLC must be correlated with the physical examination.

The clinical presentation of acute appendicitis may be atypical, imitate other disease conditions. About one-third of cases have atypical presentations. Judicious use of TLC along with other inflammatory markers and investigation with clinical correlation can have promising results.

\section{CONCLUSIONS}

In spite of advances in diagnostic and imaging techniques, the rates of the negative findings on appendicectomy have not decreased much. Clinical judgment is still the most important factor in the management of patients with suspected acute appendicitis. Routine use of CT scan or diagnostic laparoscopy for all patients who are suspected to have acute appendicitis is neither cost-effective nor safe. However, use of these two diagnostic procedures in selected controversial cases can enhance the accuracy of diagnosis, reduce the cost and reduces NAR.

Leukocytosis is not a criterion to detect acute appendicitis because of its low negative predictive value and specificity. It is still a useful investigation in decision making in doubtful cases. But clinical correlation is mandatory. Elevated TLC usually correlates with disease severity.

Authors would like to acknowledge the department of surgery of Datta Meghe Institute of Medical Sciences, Nagpur.

Financial or Other Competing Interests: None. 


\section{REFERENCES}

[1] Kamran H, Naveed D, Nazir A, et al. Role of total leukocyte count in the diagnosis of acute appendicitis. J Ayub Med Coll Abbottabad 2008;20(3):70-1.

[2] Guraya SY, Al-Tuwaijri TA, Khairy GA, et al. Validity of leukocyte count to predict severity of acute appendicitis. Saudi Med J 2005;26(12):1945-7.

[3] Wani MM, Yousaf MN, Khan MA, et al. Usefulness of the alvarado scoring system with respect to age, sex and time of presentation, with regression analysis of individual parameters. Internet J Surg 2007;11(2).

[4] Mohamed A, Bhat N. Acute appendicitis dilemma of diagnosis and management. Internet $\mathrm{J}$ Surgery 2010;23(2).

[5] Khairy G. Acute appendicitis: is removal of a normal appendix still existing and can we reduce its rate? Saudi J Gastroenterol 2009;15(3):167-70.

[6] Ahmad QA, Muneera MJ, Rasool MI. Predictive value of TLC and CRP in the diagnosis of acute appendicitis. Annals King Edward Med University 2010;16(2):116-9.

[7] Alvarado A. Inflammatory markers in acute appendicitis: are we still looking for the philosopher's stone? J Surg: JSUR-1104 2018;2018(2):1-5.
[8] Birchley D. Patients with clinical acute appendicitis should have pre-operative full blood count and Creactive protein assays. Ann $\mathrm{R}$ Coll Surg Engl 2006;88(1):27-32.

[9] Lamture YR, Ramteke H, Shinde RK, et al. Clinico sonological and laboratory co-relation with histopathology of acute appendicitis to develop new diagnostic scoring system (Yash scoring system). Int Surg J 2017;4(8):2556-64.

[10] Haa SH, Honga CK, Leea Y, et al. Clinical significance of fever and leukocytosis in diagnosis of acute appendicitis in children who visit emergency department with abdominal pain. Int J Clin Pediatr 2012;1(1):9-18.

[11] Saaiq M, Niaz-Ud-Din, Jalil A, et al. Diagnostic accuracy of leukocytosis in prediction of acute appendicitis. J Coll Physicians Surg Pak 2014;24(1):67-9.

[12] Al-Gaithy ZK. Clinical value of total white blood cells and neutrophil counts in patients with suspected appendicitis: retrospective study. World J Emerg Surg 2012;7(1):32. 